A preliminary version of this manuscript has been presented at the

6th International Conference on Tethers in Space, (12-14 June 2019, Madrid, Spain).

\title{
E-sail Attitude Control with Tether Voltage Modulation
}

\author{
Marco Bassetto*, Giovanni Mengali, Alessandro A. Quarta \\ Department of Civil and Industrial Engineering, University of Pisa, I-56122, Italy
}

\begin{abstract}
An Electric Solar Wind Sail is a propellantless propulsion system that gains thrust from the interaction of solar wind particles with a grid of long and charged tethers, which are deployed by spinning the spacecraft about its symmetry axis. In an ideal arrangement, the tethers are all stretched out and the sail takes the shape of a spoked wheel. Actually, the solar wind dynamic pressure warps the tethers and, therefore, the expressions of thrust and torque vectors are difficult to predict in analytical form. Recent works have shown that the bending of the tethers induces a disturbance torque, which can be counterbalanced through a modulation of the tether electrical voltage. Under the assumption that the Electric Solar Wind Sail behaves like an axially-symmetric rigid body, this paper proves that a modulation of the tether electrical voltage is also a feasible option for actively controlling and maintaining the spacecraft attitude. The proposed control law, which is analytically derived as a function of time and the spacecraft attitude, is validated through numerical simulations.
\end{abstract}

Keywords: Electric Solar Wind Sail, torque vector model, active attitude control

\section{Nomenclature}

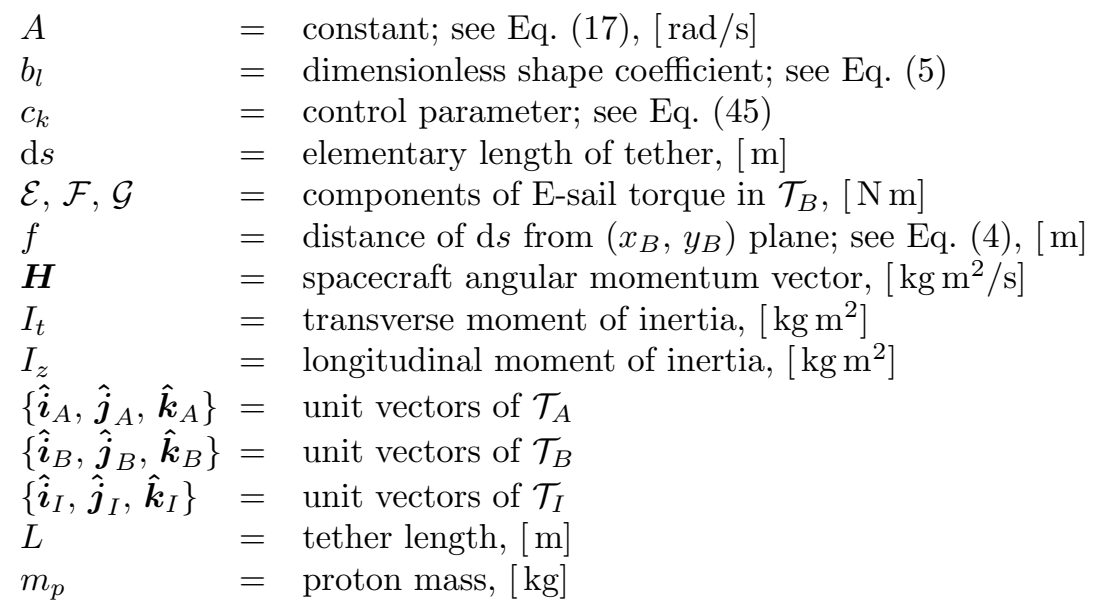

\footnotetext{
* Corresponding author

Email addresses: marco.bassetto@ing.unipi.it (Marco Bassetto), g.mengali@ing.unipi.it (Giovanni Mengali), a.quarta@ing. unipi.it (Alessandro A. Quarta) 


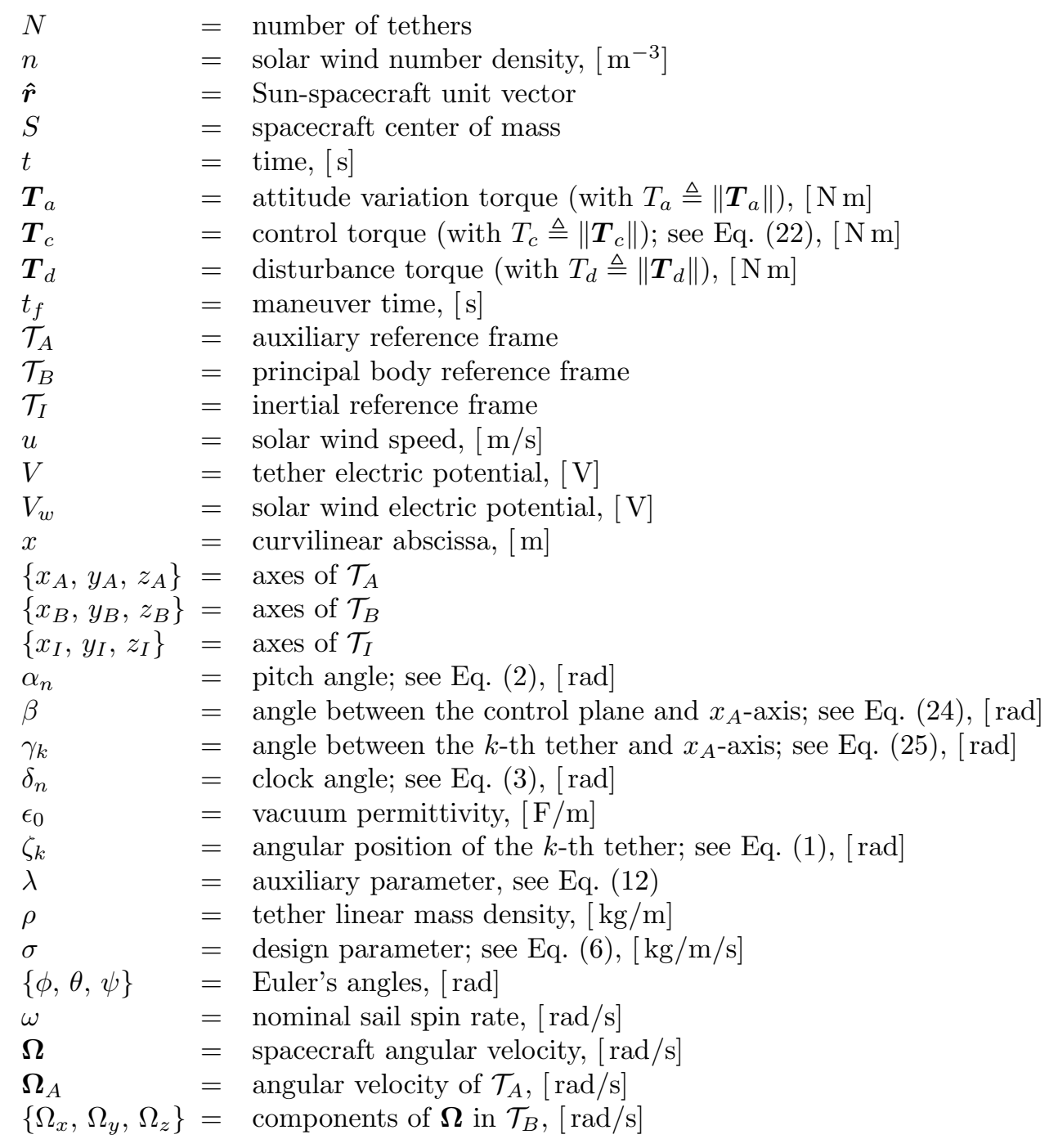

\section{Subscripts}

$\begin{array}{ll}0 & =\text { initial } \\ f & =\text { final } \\ k & =\text { referred to the } k \text {-th tether } \\ \max & =\text { maximum } \\ \min & =\text { minimum }\end{array}$

\section{$\underline{\text { Superscripts }}$}

' $=$ derivative with respect to $x$

- $\quad=$ derivative with respect to $t$

$\sim \quad=$ expected value

\section{Introduction}

An Electric Solar Wind Sail (E-sail) is a propellantless propulsion system $[1,2]$ that gains thrust from the interaction of solar wind particles with a grid of long and charged tethers, which are kept at a high voltage level by means of an electron emitter [3]. The tethers are deployed and maintained stretched by spinning the spacecraft about its symmetry axis $[4,5,6]$, so that the E-sail ideally takes the shape of a 
spoked wheel [7]; see Fig. 1. The propulsive acceleration vector may be described with the analytical model by Huo et al. [8], which incidentally shows that an E-sail does not generate any torque as long as its electrical voltage is uniform.

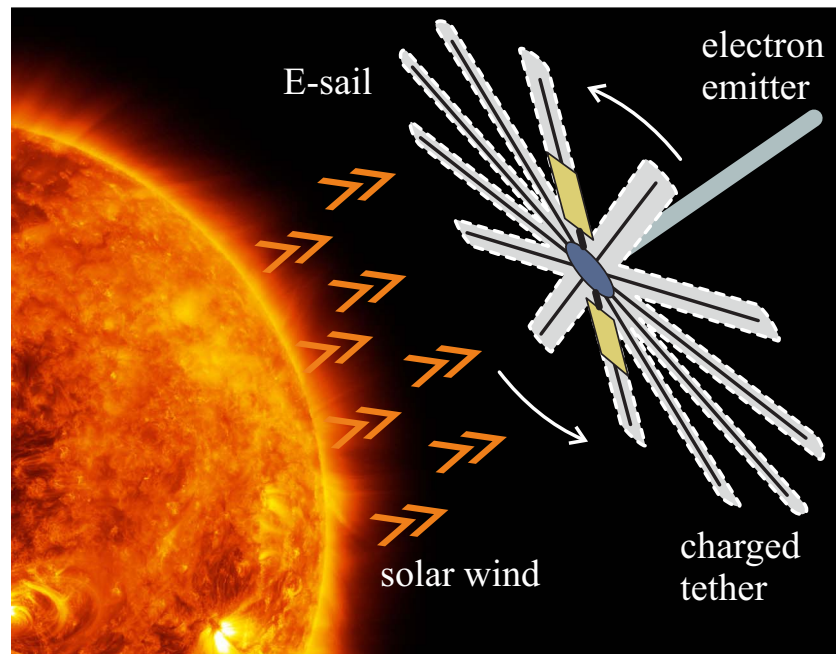

Figure 1: E-sail conceptual scheme.

The actual E-sail shape is however quite complex, as it results from the chaotic interaction between the solar wind dynamic pressure and the centrifugal force due to the spacecraft rotation. Accordingly, the tethers are not perfectly straight, and the uncertainty associated with their actual shape makes it difficult to obtain an estimate of the thrust and torque vectors, which are a necessary information for trajectory analysis and attitude control design. Recent works $[9,10]$ have addressed such a problem with the aim of obtaining approximate closed-form expressions of the E-sail thrust and torque vectors. In particular, the results of Ref. [9], based on the assumption of a Sun-facing sail [11, 12] (that is, a sail whose spin axis is aligned with the Sun-spacecraft line), show that the tether equilibrium shape is well approximated by a natural logarithmic arc when the spacecraft spin rate is sufficiently high and the electrical voltage is uniform. However, in a Sun-facing configuration the E-sail generates a purely radial thrust, while in most cases a trasverse thrust component is necessary for orbital maneuvers, since it allows the orbit angular momentum to be varied [13]. Typical advanced missions include the transfer towards linear trajectories [14], the maintenance of displaced non-Keplerian orbits $[15,16]$, interplanetary rendezvous $[17,18,19,20,21]$, or an escape from the Solar System [22, 23, 24]. A transverse thrust component may be obtained by inclining the sail nominal plane with respect to the local radial direction. However, the tether bending causes the onset of a disturbance torque [10], which induces a perturbation on the orientation of the spacecraft spin axis. The latter experiences an undamped precession-nutation motion, which eventually tends to align the thrust vector along the Sun-spacecraft direction. A possible solution to this problem is discussed in Ref. [25], where it is shown that the disturbance torque may be removed with a suitable modulation of the tether electrical voltage, without affecting the thrust vector.

The aim of this paper is to propose a new strategy for controlling and maintaining the E-sail attitude. Previous works on this subject date back to Ref. [26], in which Janhunen discussed the problem of creating and modifying the E-sail spin rate with small photonic sails applied to the tips of the main tethers. Later, Toivanen and Janhunen [27] have shown that the sail attitude can be controlled by modulating the voltage of each individual tether to produce a torque for thrust vectoring, and in Ref. [28] they have also suggested to modulate the tether voltage synchronously with the sail rotation. Finally, Janhunen and Toivanen [29] have recently presented an algorithm to control the sail attitude, in which the tethers are modeled as elastic wires, while the solar wind properties are taken from historical satellite data.

In this paper, the control is obtained by modulating the electrical voltage of each individual tether as a function of the time and the current sail attitude, assuming that the sail maintains an axially-symmetric shape. In particular, the E-sail is virtually divided in two parts, each one characterized by a precise level of 
electrical voltage. To that end, a control plane (perpendicular to the sail nominal plane) is defined [25] in order to locate where the tether voltage must change. The amplitude of the voltage modulation is obtained in an indirect way by enforcing the sail attitude to track a desired time history. The control strategy is validated through a numerical simulation, which shows that the required torque may actually be generated with a small modulation of the electrical voltage with respect to its reference value.

The paper is organized as follows. Section 2 introduces the mathematical model used to describe both the sail geometry and the disturbance torque vector. Section 3 deals with the discussion of the analytical control law, while Section 4 illustrates its effectiveness and quantifies its performance through numerical simulations. Some concluding remarks are given in the last section.

\section{Mathematical preliminaries}

To a first-order approximation, the E-sail-based spacecraft is modeled as an axially-symmetric rigid body. Its shape resembles that of a large spoked wheel, in which each spoke is substituted by a tether. All of the tethers ideally belong to the same plane, referred to as sail nominal plane. To describe the mathematical model, it is useful to introduce a principal body reference frame $\mathcal{T}_{B}\left(S ; x_{B}, y_{B}, z_{B}\right)$ of unit vectors $\left\{\hat{\boldsymbol{i}}_{B}, \hat{\boldsymbol{j}}_{B}, \hat{\boldsymbol{k}}_{B}\right\}$, where $\hat{\boldsymbol{k}}_{B}$ is aligned with the spacecraft symmetry axis, while the $\left(x_{B}, y_{B}\right)$ plane coincides with the sail nominal plane; see Fig. 2.

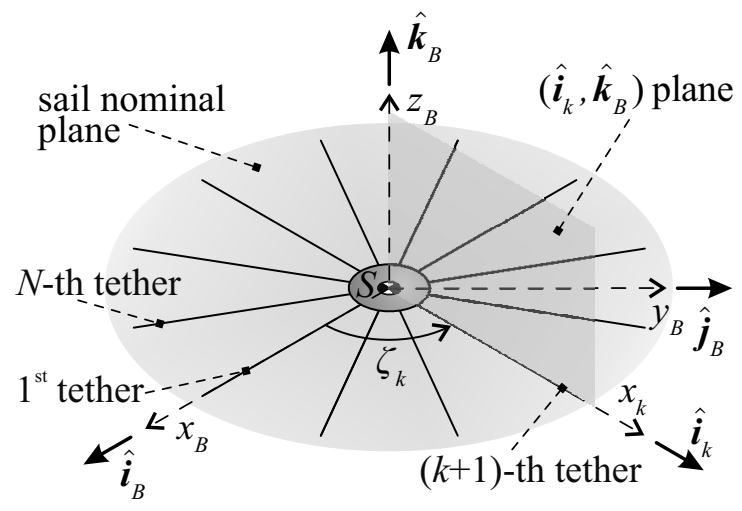

Figure 2: E-sail geometrical arrangement. Adapted from Ref. [10].

The E-sail consists of an even number $N \geq 4$ of tethers, each one denoted by a subscript $k$, with $k \in\{0,1, \ldots, N-1\}$. The angular position of the $k$-th tether, measured counterclockwise on the $\left(x_{B}, y_{B}\right)$ plane starting from $x_{B}$, is given by the azimuth angle $\zeta_{k}$ defined as

$$
\zeta_{k} \triangleq\left(\frac{2 \pi}{N}\right) k
$$

Let $\hat{\boldsymbol{r}}$ be the Sun-spacecraft unit vector, which approximately coincides with the direction of the local solar wind velocity. The spacecraft orientation is defined by the sail pitch angle $\alpha_{n} \in[0, \pi / 2] \mathrm{rad}$, that is, the angle between the direction of $\hat{\boldsymbol{r}}$ and the spacecraft symmetry axis $z_{B}$, and by the clock angle $\delta_{n} \in[0,2 \pi) \mathrm{rad}$, that is, the angle between the $x_{B}$-axis and the projection of $\hat{\boldsymbol{r}}$ on the sail nominal plane. With reference to Fig. 3 , the two attitude angles $\left\{\alpha_{n}, \delta_{n}\right\}$ are given by 


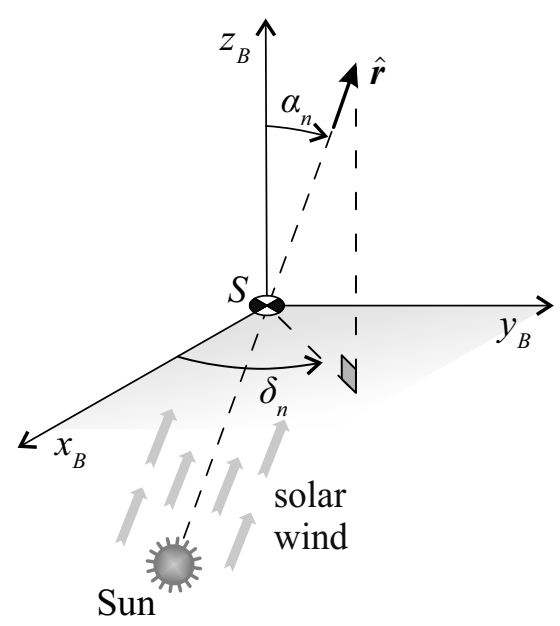

Figure 3: Pitch $\left(\alpha_{n}\right)$ and clock $\left(\delta_{n}\right)$ angles.

$$
\begin{aligned}
& \alpha_{n} \triangleq \arccos \left(\hat{\boldsymbol{r}} \cdot \hat{\boldsymbol{k}}_{B}\right) \\
& \delta_{n} \triangleq \begin{cases}\arccos \left(\frac{\hat{\boldsymbol{r}} \cdot \hat{\boldsymbol{i}}_{B}}{\left\|\hat{\boldsymbol{r}} \times \hat{\boldsymbol{k}}_{B}\right\|}\right) & \text { if }\left(\hat{\boldsymbol{r}} \cdot \hat{\boldsymbol{j}}_{B}\right) \geq 0 \\
2 \pi-\arccos \left(\frac{\hat{\boldsymbol{r}} \cdot \hat{\boldsymbol{i}}_{B}}{\left\|\hat{\boldsymbol{r}} \times \hat{\boldsymbol{k}}_{B}\right\|}\right) & \text { otherwise }\end{cases}
\end{aligned}
$$

Note that $\delta_{n}$ is undefined when $\hat{\boldsymbol{k}}_{B}$ is parallel to $\hat{\boldsymbol{r}}$ and, in that case, the pitch angle is $\alpha_{n}=0$ (that is, the spacecraft attitude is Sun-facing).

Using a more accurate description of the actual E-sail shape [9], each tether bends on the $\left(x_{k}, z_{B}\right)$ plane as a consequence of the combined effects of the centrifugal force and the solar wind dynamic pressure acting on it, as is schematically illustrated in Fig. 4. In particular, in a Sun-facing configuration the deformed tethers are well approximated by a natural logarithmic arc, whose analytical expression is

$$
f=b_{l} L \ln \left(1+\frac{x}{L}\right)
$$

where $f$ is the distance of the elementary length of tether $(\mathrm{d} s)$ from the $\left(x_{B}, y_{B}\right)$ plane, $x \in[0, L]$ is the distance of $\mathrm{d} s$ from $z_{B}$, and $L$ is the tether length; see Fig. 4.

In Eq. (4), the dimensionless shape coefficient $b_{l}$ is given by

$$
b_{l} \triangleq \frac{2 \sigma u}{\rho \omega^{2} L}
$$

where $u \simeq 400 \mathrm{~km} / \mathrm{s}$ is the average solar wind speed, $\rho \simeq 10^{-5} \mathrm{~kg} / \mathrm{m}$ is the tether linear mass density [30], while $\omega$ is the nominal sail spin rate. If the Sun-spacecraft distance is about one astronomical unit, an analytical expression for the design parameter $\sigma$ is given by $[4,27,31]$

$$
\sigma=0.18 \max \left(0, V-V_{w}\right) \sqrt{\epsilon_{0} m_{p} n}
$$

in which $V_{w}$ is the electric potential of the solar wind ions (with an average value of $1 \mathrm{kV}$ [4]), $\epsilon_{0}$ the vacuum permittivity, $m_{p}$ the proton mass, and $n$ the local solar wind number density. 


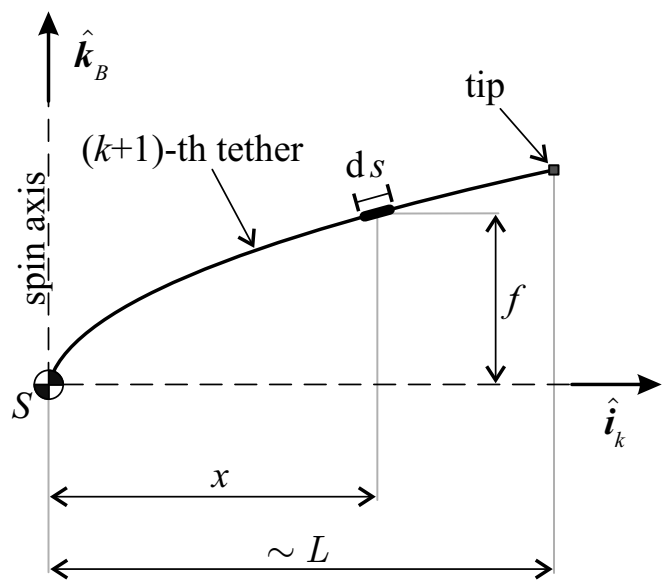

Figure 4: Generic tether deformed shape. Adapted from Ref. [25]

\subsection{Torque vector model}

The E-sail deformed shape induces the presence of a disturbance torque $\boldsymbol{T}_{d}$ to act on the spacecraft [31]. Assuming all of the tethers to have the same electrical voltage $V$, such a disturbance torque may be analytically written as $[10]$

with

$$
\boldsymbol{T}_{d}=b_{l} N L^{2} \sigma u \ln \sqrt{2}\left(\hat{\boldsymbol{k}}_{B} \times \hat{\boldsymbol{r}}\right)
$$

$$
T_{d} \triangleq\left\|\boldsymbol{T}_{d}\right\|=b_{l} N L^{2} \sigma u \ln \sqrt{2} \sin \alpha_{n}
$$

Note that the previous expression of the disturbance torque $\boldsymbol{T}_{d}$ requires the spacecraft spin rate to be sufficiently high $[9,10]$ and, as such, the tether slope to be sufficiently small, or

$$
\max \left\{\left(f^{\prime}\right)^{2}\right\}=b_{l}^{2} \simeq 0
$$

Moreover, the hypothesis of axially-symmetric E-sail is reasonable as long as its pitch angle $\alpha_{n}$ does not exceed a few degrees [25]. In that case, the disturbance torque $\boldsymbol{T}_{d}$ may be counterbalanced with a modest modulation of the tether electrical voltage $V$ by means of the design parameter $\sigma$. To that end, consider the plane, referred to as control plane, that passes through $z_{B}$ and is parallel to $\boldsymbol{T}_{d}$; see Fig. 5 . The control plane virtually splits the E-sail in two parts, characterized by two different levels of electric potential. More precisely, the tethers belonging to the half-plane containing the projection of $\hat{\boldsymbol{r}}$ are at a potential $\left(\sigma_{\max }\right)$ higher than those belonging to the other half-plane $\left(\sigma_{\min }\right)$.

In particular, the two values of the design parameter $\sigma$ are [25]

$$
\sigma_{\min }=\sigma-\frac{\Delta \sigma}{2} \quad, \quad \sigma_{\max }=\sigma+\frac{\Delta \sigma}{2}
$$

where

$$
\Delta \sigma \triangleq b_{l} \sigma N \sin (\pi / N) \ln (2) \tan \alpha_{n}
$$

As long as $\Delta \sigma / \sigma \ll 1$, the assumption of axial symmetry may be retained even when the electrical voltage of the two half-planes is (slightly) different. In that case, the required value of $\Delta \sigma$ per unit of generated torque is

$$
\lambda \triangleq \frac{\Delta \sigma}{T_{d}}=\frac{2 \sin (\pi / N)}{u L^{2} \cos \alpha_{n}}
$$

Note that $\lambda$ depends on the E-sail geometrical characteristics and on the actual sail attitude. Because $\lambda$ is independent of the E-sail shape, a control torque obtained by individually modulating the tether voltage may be generated also in the special case of a flat E-sail. The next section will use Eq. (12) to derive a control law able to change the sail attitude and, at the same time, to remove the disturbance torque due to the tether bending. 


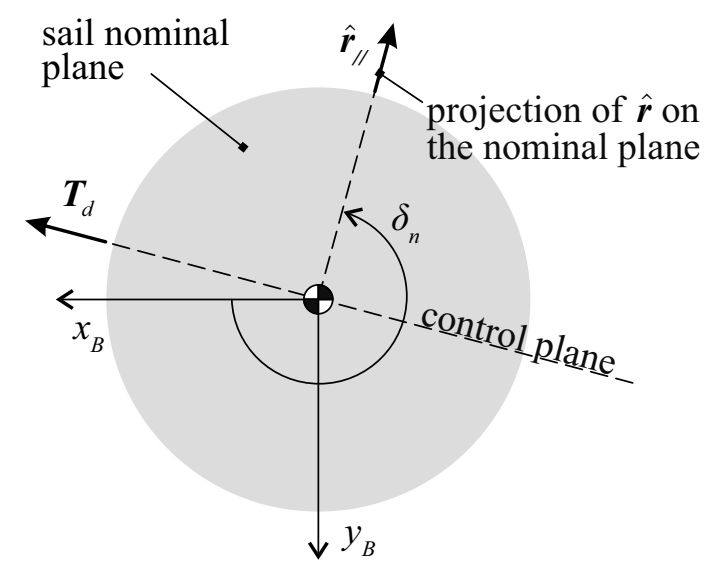

Figure 5: Disturbance torque and control plane trace.

\section{Active control law design}

The creation of a transverse thrust component requires the sail nominal plane $\left(x_{B}, y_{B}\right)$ to be oriented such as to reach a suitable value of the pitch angle $\alpha_{n}$. The aim of this section is to show how the spacecraft attitude may be adjusted with a control torque $\boldsymbol{T}_{c}$ generated through a suitable modulation of the tether electric potential. To that end, recall that the E-sail is assumed to maintain an axially-symmetric shape independent of the pitch angle $\alpha_{n}$.

The control torque $\boldsymbol{T}_{c}$ is the resultant of two vectors: 1) the attitude variation torque $\left(\boldsymbol{T}_{a}\right)$, which ensures the required change of $\alpha_{n}$ to be obtained, and 2) the vector $-\boldsymbol{T}_{d}$, which removes the disturbance torque produced by the tether bending, that is

$$
\boldsymbol{T}_{c} \triangleq \boldsymbol{T}_{a}-\boldsymbol{T}_{d}
$$

The value of $\Delta \sigma$, necessary for generating the desired control torque $\boldsymbol{T}_{c}$, is obtained from Eq. (12) as

$$
\Delta \sigma=\lambda T_{c}
$$

where $T_{c} \triangleq\left\|\boldsymbol{T}_{c}\right\|$. The two vectors on the right hand side of Eq. (13) will now be analyzed separately.

Consider first the attitude variation torque $\boldsymbol{T}_{a}$. Its magnitude $T_{a}$ is found by enforcing a desired time evolution of the sail pitch angle. Without loss of generality, assume that the E-sail is initially placed in a Sun-facing configuration, such that its nominal plane $\left(x_{B}, y_{B}\right)$ is orthogonal to the Sun-sail direction; see Fig. 3. The attitude maneuver is better visualized with the aid of two additional reference frames. More precisely, let $\mathcal{T}_{I}\left(S ; x_{I}, y_{I}, z_{I}\right)$ be an inertial reference frame of unit vectors $\left\{\hat{\boldsymbol{i}}_{I}, \hat{\boldsymbol{j}}_{I}, \hat{\boldsymbol{k}}_{I}\right\}$ with $\hat{\boldsymbol{i}}_{I} \equiv \hat{\boldsymbol{i}}_{B_{0}}$ and $\hat{\boldsymbol{k}}_{I} \equiv \hat{\boldsymbol{k}}_{B_{0}} \equiv \hat{\boldsymbol{r}}$, where $\hat{\boldsymbol{i}}_{B_{0}}\left(\right.$ or $\left.\hat{\boldsymbol{k}}_{B_{0}}\right)$ is the unit vector $\hat{\boldsymbol{i}}_{B}$ (or $\hat{\boldsymbol{k}}_{B}$ ) at the initial time $t=0$. Introduce also an auxiliary reference frame $\mathcal{T}_{A}\left(S ; x_{A}, y_{A}, z_{A}\right)$ of unit vectors $\left\{\hat{\boldsymbol{i}}_{A}, \hat{\boldsymbol{j}}_{A}, \hat{\boldsymbol{k}}_{A}\right\}$ with $\hat{\boldsymbol{j}}_{A} \equiv \hat{\boldsymbol{j}}_{I}$. In particular, $\mathcal{T}_{A}$ is a non-inertial reference frame, which rotates about the $y_{A}$-axis (fixed in the inertial space) of an angle equal to the pitch angle $\alpha_{n}$, until the attitude maneuver is completed; see the sketch in Fig. 6.

The rotation of the sail nominal plane $\left(x_{B}, y_{B}\right)$ is chosen to track a desired time variation of the pitch angle, that is

$$
\dot{\widetilde{\alpha}}_{n} \triangleq A\left[1-\frac{4}{t_{f}^{2}}\left(t-\frac{t_{f}}{2}\right)^{2}\right]\left[1(t)-1\left(t-t_{f}\right)\right]
$$

where the symbol $\widetilde{\square}$ denotes the desired value, $1(t)$ is the step function, $t_{f}$ the attitude maneuver time, and $A$ is a suitable constant. Note that the initial and final equilibrium conditions at the beginning and at the end of the attitude maneuver, that is, $\dot{\widetilde{\alpha}}_{n}(0)=\dot{\widetilde{\alpha}}_{n}\left(t_{f}\right)=0$, are automatically met. Equation (15) can be integrated over time to get

$$
\widetilde{\alpha}_{n}=\frac{2 A}{t_{f}}\left(t^{2}-\frac{2}{3} \frac{t^{3}}{t_{f}}\right)\left[1(t)-1\left(t-t_{f}\right)\right]
$$




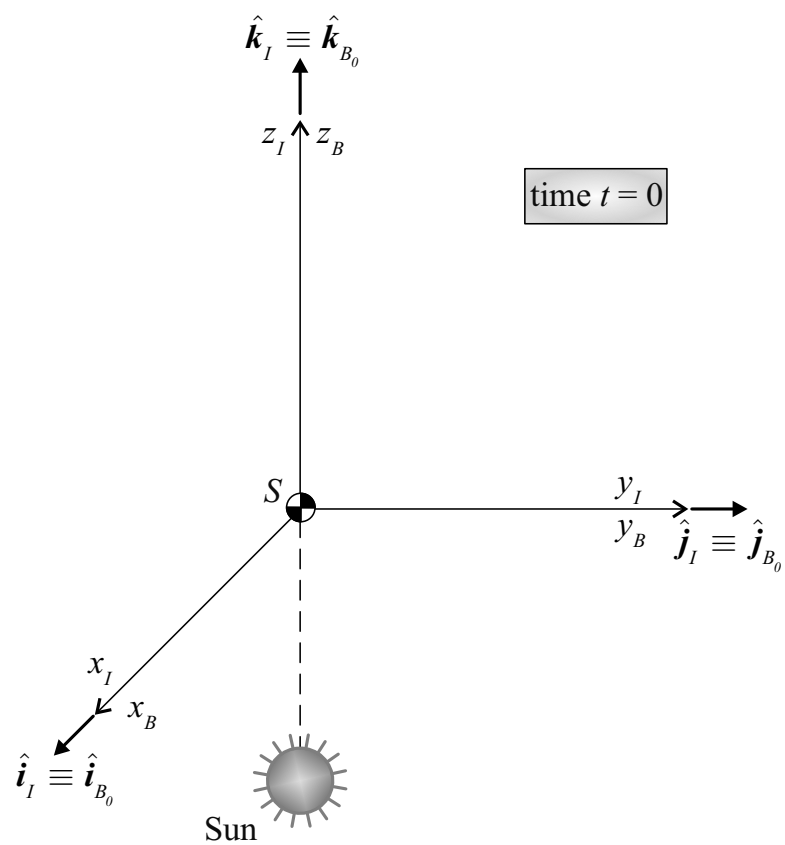

(a) E-sail orientation at $t=0$.

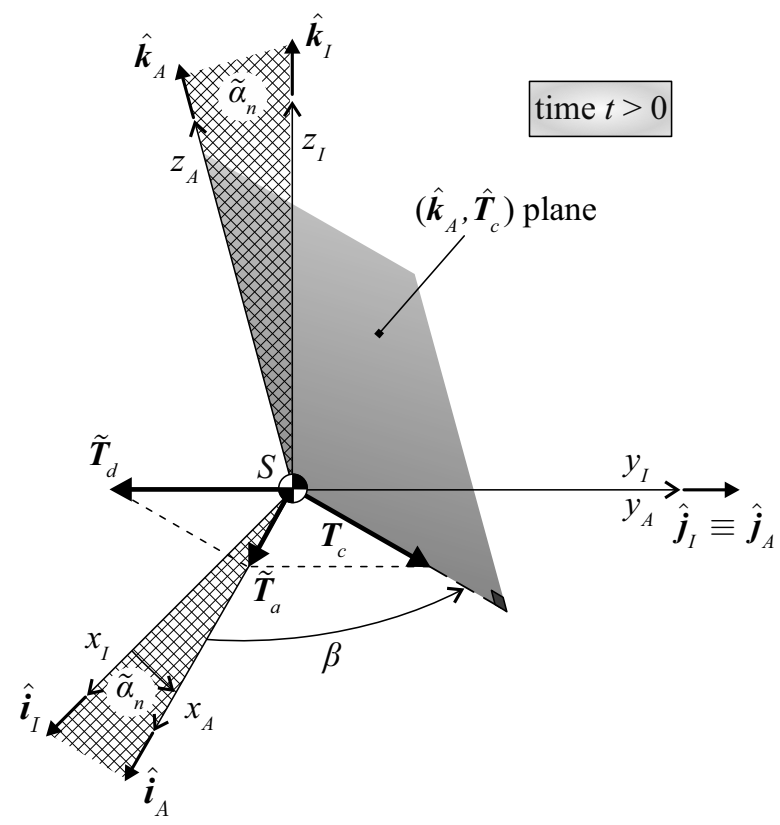

(b) Attitude maneuver.

Figure 6: Sketch of the attitude maneuver with the control torque.

where the constant $A$ is obtained by enforcing the final condition $\widetilde{\alpha}_{n}\left(t_{f}\right) \triangleq \widetilde{\alpha}_{n_{f}}$, viz.

$$
A=\frac{3 \widetilde{\alpha}_{n_{f}}}{2 t_{f}}
$$

from which

$$
\dot{\widetilde{\alpha}}_{n}=\frac{6 \widetilde{\alpha}_{n_{f}}}{t_{f}}\left[\frac{t}{t_{f}}-\left(\frac{t}{t_{f}}\right)^{2}\right]\left[1(t)-1\left(t-t_{f}\right)\right]
$$


Figure 7 shows the desired time variation of the pitch angle and its derivative, corresponding to Eqs. (16) and (18), respectively.
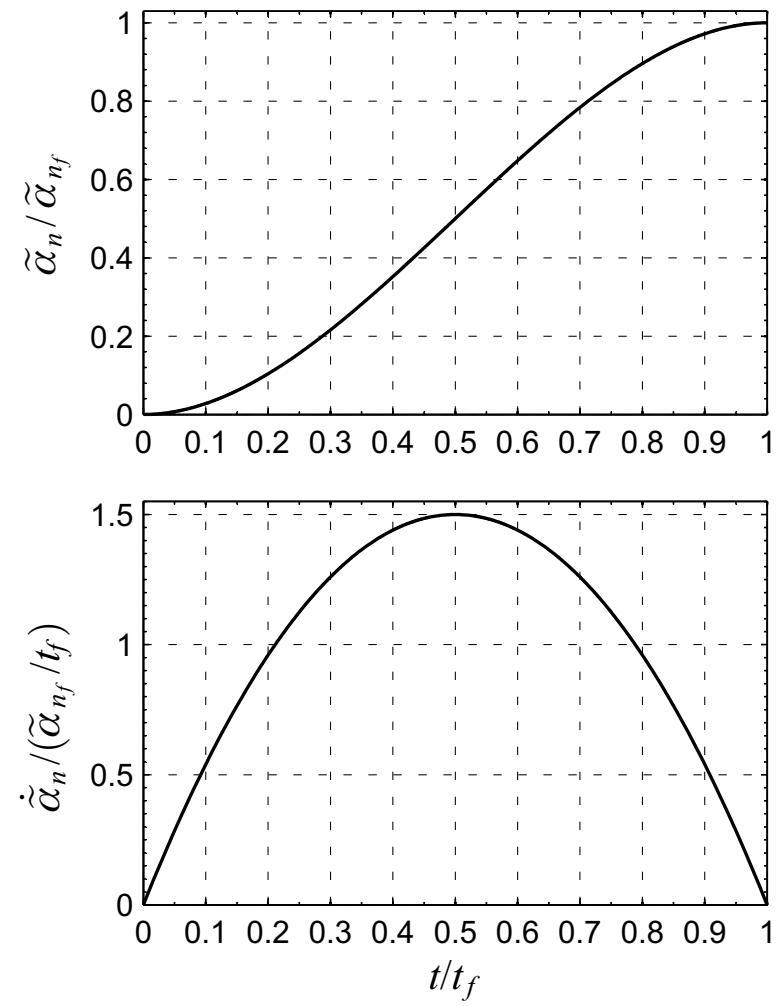

Figure 7: Desired time variation of the pitch angle during the E-sail attitude maneuver.

The attitude variation torque $\boldsymbol{T}_{a}$ is chosen such that the rate of change of $\alpha_{n}$ equals that given by Eq. (18). Let $\boldsymbol{\Omega}_{A}=\dot{\tilde{\alpha}}_{n} \hat{\boldsymbol{j}}_{A}$ be the angular velocity with which the auxiliary frame $\mathcal{T}_{A}$ rotates about its $y_{A}$-axis. If the pitch angle were able to meet the desired time variation of Eq. (16), the principal body frame would exactly follow the auxiliary frame during the whole attitude maneuver and, accordingly, the E-sail spin axis $z_{B}$ would always coincide with $z_{A}$. The desired spacecraft angular momentum vector $\widetilde{\boldsymbol{H}}$ in the auxiliary frame is therefore $\widetilde{\boldsymbol{H}}=I_{z} \omega \hat{\boldsymbol{k}}_{A}$, where $I_{z}$ is the spacecraft longitudinal moment of inertia. As a result, the required attitude variation torque $\widetilde{\boldsymbol{T}}_{a}$ is given by

$$
\widetilde{\boldsymbol{T}}_{a}=\boldsymbol{\Omega}_{A} \times \widetilde{\boldsymbol{H}}=T_{a} \hat{\boldsymbol{i}}_{A}
$$

where

$$
T_{a} \triangleq I_{z} \omega \dot{\widetilde{\alpha}}_{n}=\frac{6 \widetilde{\alpha}_{n_{f}} I_{z} \omega}{t_{f}}\left[\frac{t}{t_{f}}-\left(\frac{t}{t_{f}}\right)^{2}\right]\left[1(t)-1\left(t-t_{f}\right)\right]
$$

Moreover, under the previous assumption that $z_{B} \equiv z_{A}$, the onset of a pitch angle different from zero also causes a disturbance torque to act along the $y_{A}$-axis, that is, $\widetilde{\boldsymbol{T}}_{d}=-T_{d} \hat{\boldsymbol{j}}_{A}$, where $T_{d}$ is given by Eq. (8). From Eq. (13), the control torque is

$$
\boldsymbol{T}_{c} \simeq \widetilde{\boldsymbol{T}}_{a}-\widetilde{\boldsymbol{T}}_{d}
$$

or

$$
\boldsymbol{T}_{c} \simeq \frac{6 \widetilde{\alpha}_{n_{f}} I_{z} \omega}{t_{f}}\left[\frac{t}{t_{f}}-\left(\frac{t}{t_{f}}\right)^{2}\right]\left[1(t)-1\left(t-t_{f}\right)\right] \hat{\boldsymbol{i}}_{A}+\frac{N L(\sigma u)^{2} \ln 2}{\rho \omega^{2}} \sin \alpha_{n} \hat{\boldsymbol{j}}_{A}
$$

While the attitude variation torque $\widetilde{\boldsymbol{T}}_{a}$ is an explicit function of time, $\widetilde{\boldsymbol{T}}_{d}$ depends on $\alpha_{n}$, which must therefore be measured during the attitude maneuver. Because $\widetilde{\boldsymbol{T}}_{a}$ and $\widetilde{\boldsymbol{T}}_{d}$ are perpendicular to each other, 
the magnitude of the control torque is

$$
T_{c} \triangleq\left\|\boldsymbol{T}_{c}\right\|=\sqrt{\frac{36 \widetilde{\alpha}_{n_{f}}^{2} I_{z}^{2} \omega^{2}}{t_{f}^{2}}\left[\frac{t}{t_{f}}-\left(\frac{t}{t_{f}}\right)^{2}\right]^{2}\left[1(t)-1\left(t-t_{f}\right)\right]+\frac{N^{2} L^{2}(\sigma u)^{4}(\ln 2)^{2}}{\rho^{2} \omega^{4}} \sin ^{2} \alpha_{n}}
$$

In analogy with the previously described approach for counterbalancing the disturbance torque alone, the sail nominal plane is now split in two parts by the control plane, which is orthogonal to the $\left(x_{A}, y_{A}\right)$ plane and passes through $\boldsymbol{T}_{c}$. Again, the tethers belonging to the half-planes separated by the control plane are set to two different levels of electric potential, that is, according to Eq. (14), $\sigma \pm \lambda T_{c} / 2$. The orientation of the control plane is defined by the angle $\beta=\beta\left(t, \alpha_{n}\right) \in[0,2 \pi) \mathrm{rad}$, which is the angle between the direction of $\boldsymbol{T}_{c}$ and the $x_{A}$-axis, measured counterclockwise from $x_{A}$. With reference to Fig. 8 , the angle $\beta$ is obtained as

$$
\beta \triangleq \arctan \left(\frac{T_{d}}{T_{a}}\right)
$$

where $T_{d}$ and $T_{a}$ are given by Eqs. (8) and (20), respectively.

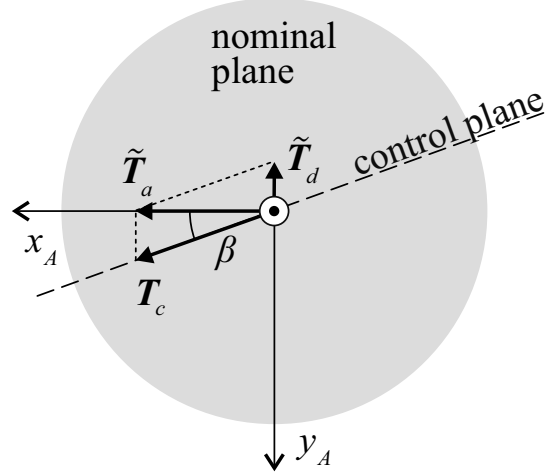

Figure 8: Control torque and orientation of the control plane trace.

Note that when the attitude maneuver ends, $T_{a} \rightarrow 0$ and $\beta \rightarrow \pi / 2 \mathrm{rad}$. The value of $\sigma_{k}$ to be assigned to each tether depends on the angular displacement $\gamma_{k}$ of the generic tether relative to the control plane, that is

$$
\gamma_{k} \triangleq \omega t+\zeta_{k}-\beta
$$

The resultant control law is therefore

$$
\sigma_{k}=\left\{\begin{array}{ccc}
\sigma-\frac{\lambda T_{c}}{2} & \text { if } & \gamma_{k} \in[0, \pi) \mathrm{rad} \\
\sigma+\frac{\lambda T_{c}}{2} & \text { if } & \gamma_{k} \in[\pi, 2 \pi) \mathrm{rad}
\end{array}\right.
$$

where $\sigma$ and $\lambda$ are given by Eqs. (6) and (12), respectively, while $T_{c}$ must be computed from Eq. (23). Finally, $\beta$ is obtained as

$$
\beta= \begin{cases}\arctan \left(\frac{T_{d}}{T_{a}}\right) & \text { if } t \leq t_{f} \\ \frac{\pi}{2} \mathrm{rad} & \text { if } t>t_{f}\end{cases}
$$

\section{Numerical simulations}

The effectiveness of the proposed control law is now checked by numerically integrating the Euler's attitude equations. To that end, the torque components generated by the E-sail are written in the principal 
body frame $\mathcal{T}_{B}$. Let $\{\phi, \theta, \psi\}$ be the Euler's angles that define the orientation of $\mathcal{T}_{B}$ with respect to the inertial reference frame $\mathcal{T}_{I}$. Using the rotational sequence $3(\psi) \rightarrow 1(\phi) \rightarrow 2(\theta)[32]$ to describe the orientation of $\mathcal{T}_{B}$ relative to $\mathcal{T}_{I}$, the kinematic equations of a rigid E-sail-based spacecraft are [25]

$$
\begin{aligned}
& \dot{\phi}=\Omega_{x} \cos \theta+\Omega_{z} \sin \theta \\
& \dot{\theta}=\Omega_{y}-\left(\Omega_{z} \cos \theta-\Omega_{x} \sin \theta\right) \tan \phi \\
& \dot{\psi}=\left(\Omega_{z} \cos \theta-\Omega_{x} \sin \theta\right) \sec \phi
\end{aligned}
$$

where $\left\{\Omega_{x}, \Omega_{y}, \Omega_{z}\right\}$ are the components of the spacecraft angular velocity $\Omega$ in $\mathcal{T}_{B}$. Note that the pitch and clock angles are related to the Euler's angles as

$$
\begin{aligned}
& \cos \alpha_{n}=\cos \phi \cos \theta \\
& \sin \alpha_{n} \sin \delta_{n}=\sin \phi \\
& \sin \alpha_{n} \cos \delta_{n}=-\cos \phi \sin \theta
\end{aligned}
$$

Accordingly, the Euler's equations may be written as [25]

$$
\begin{aligned}
& \dot{\Omega}_{x}=\frac{I_{t}-I_{z}}{I_{t}} \Omega_{y} \Omega_{z}+\frac{\mathcal{E}}{I_{t}} \\
& \dot{\Omega}_{y}=\frac{I_{z}-I_{t}}{I_{t}} \Omega_{x} \Omega_{z}+\frac{\mathcal{F}}{I_{t}} \\
& \dot{\Omega}_{z}=\frac{\mathcal{G}}{I_{z}}
\end{aligned}
$$

where $I_{t}$ is the spacecraft transverse moment of inertia, while $\{\mathcal{E}, \mathcal{F}, \mathcal{G}\}$ are the components of the total torque generated by the E-sail in the principal body frame $\mathcal{T}_{B}$. Note that $\{\mathcal{E}, \mathcal{F}, \mathcal{G}\}$ include not only the contribution to the total torque given by the modulation of the electrical voltage, but also the effects of the tether bending. In particular, according to Ref. [25], the components of the total torque can be written as

$$
\mathcal{E} \triangleq \sum_{k=0}^{N-1} \mathcal{E}_{k} \quad, \quad \mathcal{F} \triangleq \sum_{k=0}^{N-1} \mathcal{F}_{k} \quad, \quad \mathcal{G} \triangleq \sum_{k=0}^{N-1} \mathcal{G}_{k}
$$

where

$$
\mathcal{E}_{k} \triangleq \int_{0}^{L} \mathrm{~d} \mathcal{E}_{k} \quad, \quad \mathcal{F}_{k} \triangleq \int_{0}^{L} \mathrm{~d} \mathcal{F}_{k} \quad, \quad \mathcal{G}_{k} \triangleq \int_{0}^{L} \mathrm{~d} \mathcal{G}_{k}
$$

are the components of the total torque provided by the $k$-th tether. Bearing in mind that $b_{l}^{2} \simeq 0$ (see Eq.(9)), the complete expressions of $\mathrm{d} \mathcal{E}_{k}, \mathrm{~d} \mathcal{F}_{k}$, and $\mathrm{d} \mathcal{G}_{k}$, taken from Ref. [25], are reported below for the sake of completeness

$$
\begin{aligned}
\mathrm{d} \mathcal{E}_{k} & =\sigma_{k} u\left[\left(f-x f^{\prime}\right) \sin \zeta_{k} \sin \alpha_{n} \cos \left(\delta_{n}-\zeta_{k}\right)-f \sin \alpha_{n} \sin \delta_{n}+x \sin \zeta_{k} \cos \alpha_{n}\right] \mathrm{d} x \\
\mathrm{~d} \mathcal{F}_{k} & =\sigma_{k} u\left[\left(x f^{\prime}-f\right) \cos \zeta_{k} \sin \alpha_{n} \cos \left(\delta_{n}-\zeta_{k}\right)+f \sin \alpha_{n} \cos \delta_{n}-x \cos \zeta_{k} \cos \alpha_{n}\right] \mathrm{d} x \\
\mathrm{~d} \mathcal{G}_{k} & =\sigma_{k} u x \sin \alpha_{n} \sin \left(\delta_{n}-\zeta_{k}\right) \mathrm{d} x
\end{aligned}
$$

The torque components from the $k$-th tether are obtained by integrating Eqs. (39)-(41) along the tether 
length. The result is

$$
\begin{aligned}
& \mathcal{E}_{k}=c_{k}\left\{b_{l} \sin \alpha_{n}\left[\cos \left(\delta_{n}-\zeta_{k}\right) \sin \zeta_{k}(\ln 8-2)-\sin \delta_{n}(\ln 4-1)\right]+\frac{\cos \alpha_{n} \sin \zeta_{k}}{2}\right\} \\
& \mathcal{F}_{k}=c_{k}\left\{b_{l} \sin \alpha_{n}\left[\cos \delta_{n}(\ln 4-1)-\cos \left(\delta_{n}-\zeta_{k}\right) \cos \zeta_{k}(\ln 8-2)\right]-\frac{\cos \alpha_{n} \cos \zeta_{k}}{2}\right\} \\
& \mathcal{G}_{k}=c_{k}\left\{\frac{\sin \left(\delta_{n}-\zeta_{k}\right) \sin \alpha_{n}}{2}\right\}
\end{aligned}
$$

where

$$
c_{k} \triangleq u L^{2} \sigma_{k}
$$

The special case of flat E-sail can be retrieved by simply setting $b_{l}=0$.

For exemplary purposes assume that $I_{z}=3 I_{t}=3000 \mathrm{~kg} \mathrm{~m}^{2}, L=2 \mathrm{~km}$, and $\omega \simeq 0.0758 \mathrm{rad} / \mathrm{s}$, from which $b_{l} \simeq 6.471 \times 10^{-3}$. The initial conditions are $\phi(0)=\theta(0)=\psi(0)=\Omega_{x}(0)=\Omega_{y}(0)=0$ and $\Omega_{z}(0)=\omega$. Equations (28)-(30) and (34)-(36) have been numerically integrated by implementing the control law described by Eqs. (26) and (27) with $\widetilde{\alpha}_{n_{f}}=5 \mathrm{deg}$ and $t_{f}=2$ minutes. Two E-sail configurations have been considered, the former with $N=16$, the latter with $N=32$. In both cases, the simulations are reported in a time span greater than $t_{f}$ to verify whether the E-sail is able to maintain its final attitude with the proposed control law.

The results are shown in Figs. 9-12. The time evolutions of the sail pitch angle during and after the attitude maneuver (see Fig. 9) exhibit an oscillatory behavior around a value close to the desired angle of 5 deg. It can also be observed that the mean asymptotic values tend to slightly decrease over time. Such a behavior is due to the combined effect of the torque component generated along the $z_{B}$-axis (see Fig. 10), which causes a small increase of the sail spin rate from $0.0758 \mathrm{rad} / \mathrm{s}$ to $0.0761 \mathrm{rad} / \mathrm{s}$, and the presence of the disturbance torque $\boldsymbol{T}_{d}$. As a matter of fact, the observed decrease of $\alpha_{n}$ no longer occurs when the torque along $z_{B}$ is set equal to zero (i.e., $\mathcal{G}=0$ ) and/or when the E-sail takes a perfectly flat shape (i.e., $b_{l}=0$ ). This is clearly illustrated in Fig. 11, where the time evolution of $\alpha_{n}$ is shown for $N=16$ in three different cases of $\left\{b_{l} \neq 0, \mathcal{G} \neq 0\right\},\left\{b_{l}=0, \mathcal{G} \neq 0\right\}$, and $\left\{b_{l} \neq 0, \mathcal{G}=0\right\}$. Figure 10 also shows the components $\mathcal{E}$ and $\mathcal{F}$, whose values never exceed $0.25 \mathrm{Nm}$. Finally, Fig. 12 reports the time evolutions of $\Delta \sigma / \sigma$. Note that $\Delta \sigma=0$ at $t=0$ because of the assumption that the E-sail starts its attitude maneuver from a Sun-facing configuration. During the attitude variation, the maximum value of $\Delta \sigma / \sigma$ is about 0.0651 (or 0.0327 ) when $N=16$ (or $N=32$ ). Therefore, for both E-sail configurations, the electrical voltage modulation is a few percent only of its nominal value. The values of $\Delta \sigma$ for $t>t_{f}$ allow an E-sail to maintain its final attitude, in accordance with the results presented in Ref. [25].

For a given E-sail configuration, it is interesting to compare the order of magnitude of $\Delta \sigma$ required for varying the spacecraft attitude with that necessary for maintaining it. To that end, since

$$
\lambda T_{a} \propto \sin \left(\frac{\pi}{N}\right) \quad, \quad \lambda T_{d} \propto N \sin \left(\frac{\pi}{N}\right)
$$

it turns out that $T_{a} / T_{d} \propto N^{-1}$. This is confirmed by the results shown in Fig. 12, in which the required $\Delta \sigma$ during the attitude maneuver is maximum in the configuration with the smallest number of tethers $(N=16)$. In other terms, when $N$ is sufficiently small, the voltage modulation necessary for removing the disturbance torque becomes negligible when compared to that required for changing the spacecraft attitude. In those cases, the assumption of a flat sail (corresponding to negligible disturbance torques due to the tether bending) becomes a reasonable approximation, useful for simplifying the mission analysis.

\section{Conclusions}

The attitude of an E-sail-based spacecraft may be changed by modulating the electrical voltage of each tether. To that end, an analytical control law has been proposed, in which the voltage level of the generic tether is expressed as a function of the time and the sail pitch angle. A small variation of the voltage level is 


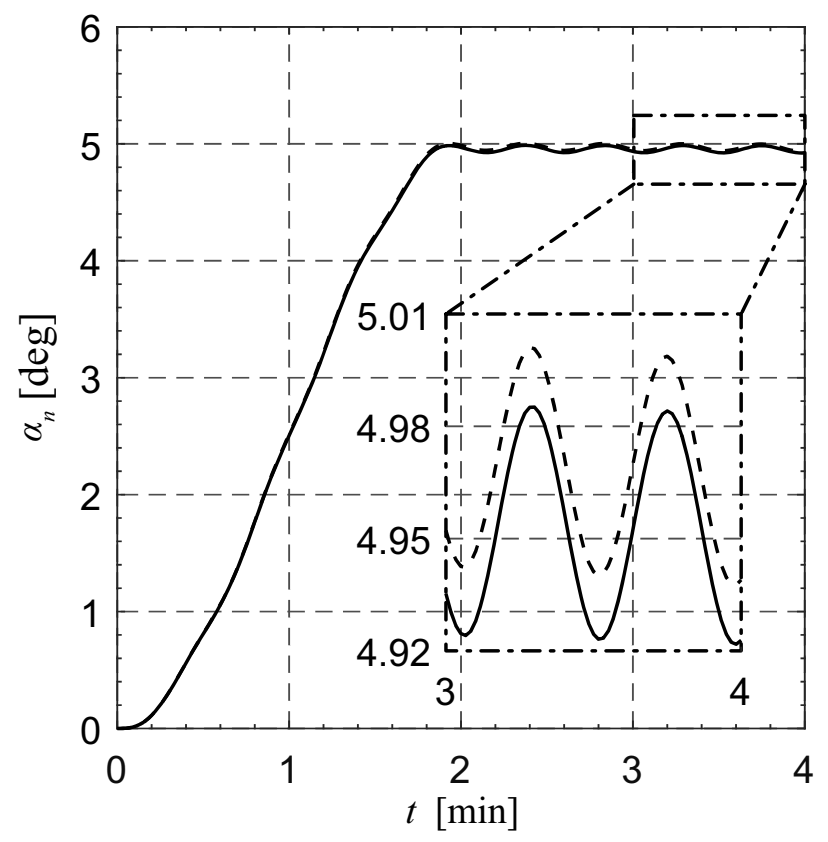

Figure 9: Time evolution of the pitch angle $\alpha_{n}$ during attitude maneuver when $N=16$ (solid line) and $N=32$ (dotted line).

sufficient for most practical purposes. The proposed method for controlling the spacecraft attitude is easy to implement and offers good performance in terms of attitude reorientation time.

The numerical simulations have shown that the attitude change induces the onset of a torque along the spacecraft symmetry axis, which is responsible (together with the disturbance torque due to the tether bending) of a small variation of the sail spin rate. As a result, at the end of the attitude maneuver, the pitch angle is not maintained at a constant value, but tends to slowly decrease over time. Such an unwanted phenomenon is instead absent in the case when the torque along the symmetry axis is set equal to zero, or the E-sail shape is assumed to be flat. Moreover, when the E-sail is made of a small number of tethers, the control torque necessary for maintaining the final attitude is small compared to the one required for attitude variation. In those cases, the approximation of flat sail becomes accurate and useful for greatly simplifying the mission analysis, allowing more refined control laws to be derived. The problem of maintaining the target attitude with a higher level of accuracy is currently under development, and represents a natural extension of this work.

\section{References}

[1] P. Janhunen, Electric sail for spacecraft propulsion, Journal of Propulsion and Power 20 (4) (2004) 763-764, doi: $10.2514 / 1.8580$

[2] P. Janhunen, A. Sandroos, Simulation study of solar wind push on a charged wire: basis of solar wind electric sail propulsion, Annales Geophysicae 25 (3) (2007) 755-767, doi: 10.5194/angeo-25-755-2007.

[3] P. Janhunen, Status report of the electric sail in 2009, Acta Astronautica 68 (5-6) (2011) 567-570, doi: 10.1016/j.actaastro.2010.02.007.

[4] P. Janhunen, P. K. Toivanen, J. Polkko, S. Merikallio, P. Salminen, E. Haeggström, H. Seppänen, R. Kurppa, J. Ukkonen, S. Kiprich, G. Thornell, H. Kratz, L. Richter, O. Krömer, R. Rosta, M. Noorma, J. Envall, S. Lätt, G. Mengali, A. A. Quarta, H. Koivisto, O. Tarvainen, T. Kalvas, J. Kauppinen, A. Nuottajärvi, A. Obraztsov, Invited article: Electric solar wind sail: toward test missions, Review of Scientific Instruments 81 (11) (2010) 111301-1-11301-11, doi: $10.1063 / 1.3514548$.

[5] P. Janhunen, A. A. Quarta, G. Mengali, Electric solar wind sail mass budget model, Geoscientific Instrumentation, Methods and Data Systems 2 (1) (2013) 85-95, doi: 10.5194/gi-2-85-2013.

[6] J. Fulton, H. Schaub, Fixed-axis electric sail deployment dynamics analysis using hub-mounted momentum control, Acta Astronautica 144 (2018) 160-170, doi: 10.1016/j.actaastro.2017.11.048. 

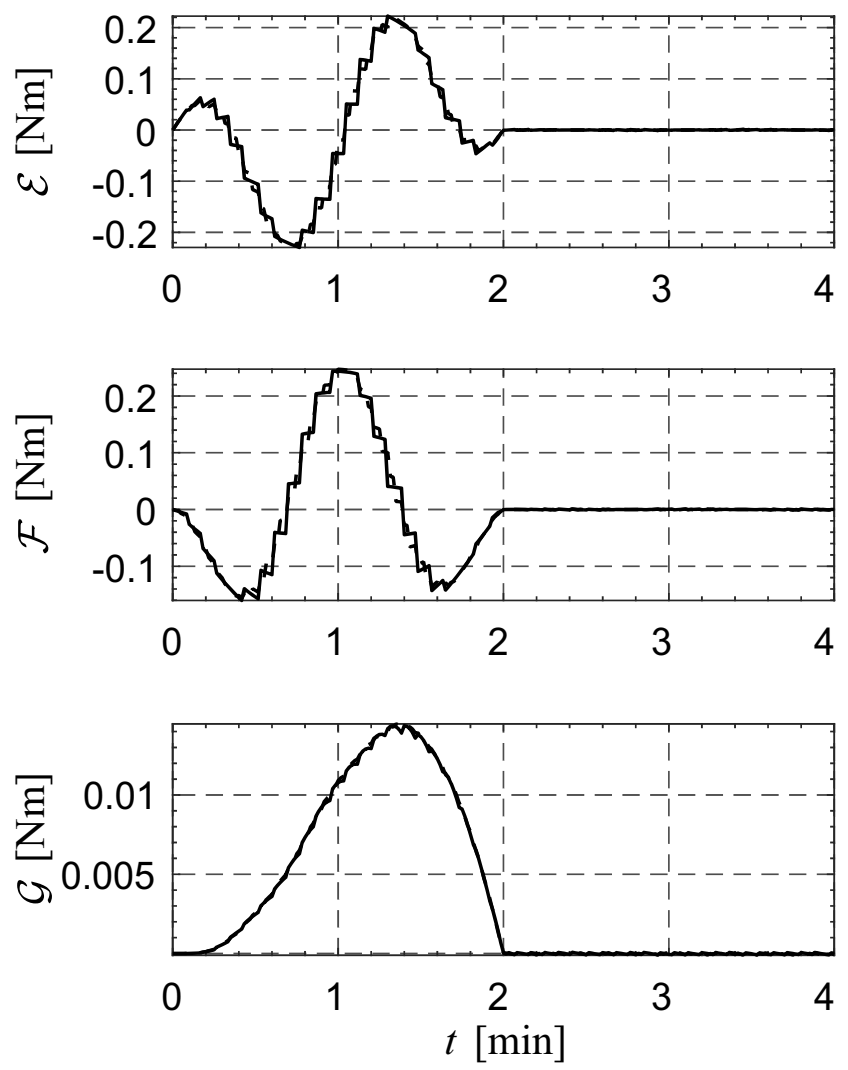

Figure 10: Components of E-sail torque in $\mathcal{T}_{B}$ during attitude maneuver when $N=16$ (solid line) and $N=32$ (dotted line).

[7] A. A. Quarta, G. Mengali, Minimum-time trajectories of electric sail with advanced thrust model, Aerospace Science and Technology 55 (2016) 419-430, doi: 10.1016/j.ast.2016.06.020.

[8] M. Huo, G. Mengali, A. A. Quarta, Electric sail thrust model from a geometrical perspective, Journal of Guidance, Control, and Dynamics 41 (3) (2018) 735-741, doi: 10.2514/1.G003169.

[9] M. Bassetto, G. Mengali, A. A. Quarta, Thrust and torque vector characteristics of axially-symmetric E-sail, Acta Astronautica 146 (2018) 134-143, doi: 10.1016/j.actaastro.2018.02.035.

[10] M. Bassetto, G. Mengali, A. A. Quarta, Stability and control of spinning E-sail in heliostationary orbit, Journal of Guidance, Control, and Dynamics 42 (2) (2018) 425-431, doi: 10.2514/1.G003788.

[11] G. Mengali, A. A. Quarta, G. Aliasi, A graphical approach to electric sail mission design with radial thrust, Acta Astronautica 82 (2) (2013) 197-208, doi: 10.1016/j.actaastro.2012.03.022.

[12] A. A. Quarta, G. Mengali, Analysis of electric sail heliocentric motion under radial thrust, Journal of Guidance, Control, and Dynamics 39 (6) (2016) 1431-1435, doi: 10.2514/1.G001632.

[13] M. Bassetto, A. A. Quarta, G. Mengali, Locally-optimal electric sail transfer, Proceedings of the Institution of Mechanical Engineers, Part G: Journal of Aerospace Engineering, 233 (1) (2019) 166-179, doi: 10.1177/0954410017728975.

[14] A. A. Quarta, G. Mengali, Optimal solar sail transfer to linear trajectories, Acta Astronautica 82 (2) (2013) 189-196, doi: 10.1016/j.actaastro.2012.03.005.

[15] L. Niccolai, A. A. Quarta, G. Mengali, Electric sail-based displaced orbits with refined thrust model, Proceedings of the Institution of Mechanical Engineers, Part G: Journal of Aerospace Engineering 232 (3) (2018) 423-432, doi: $10.1177 / 0954410016679195$.

[16] L. Niccolai, A. A. Quarta, G. Mengali, Electric sail elliptic displaced orbits with advanced thrust model, Acta Astronautica 138 (2017) 503-511, doi: $10.1016 / \mathrm{j}$.actaastro.2016.10.036.

[17] A. A. Quarta, G. Mengali, Electric sail missions to potentially hazardous asteroids, Acta Astronautica 66 (9-10) (2010) 1506-1519, doi: 10.1016/j.actaastro.2009.11.021.

[18] G. Mengali, A. A. Quarta, Optimal nodal flyby with near-Earth asteroids using electric sail, Acta Astronautica 104 (2) (2014) 450-457, doi: 10.1016/j.actaastro.2014.02.012.

[19] A. A. Quarta, G. Mengali, P. Janhunen, Electric sail option for cometary rendezvous, Acta Astronautica 127 (2016) 684-692, doi: 10.1016/j.actaastro.2016.06.020. 


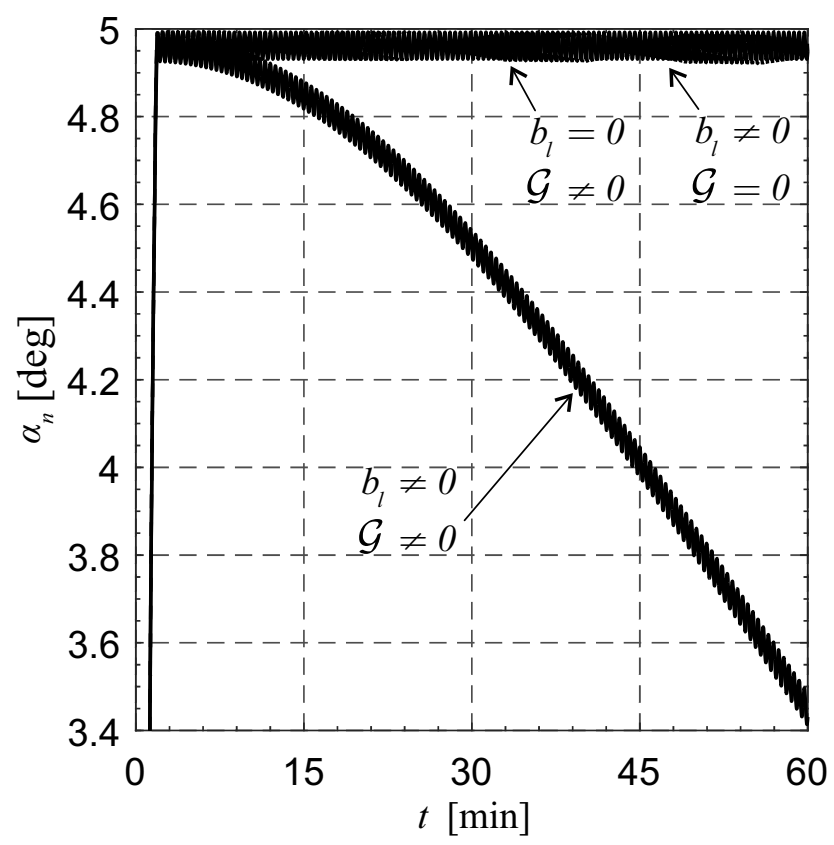

Figure 11: Time evolution of $\alpha_{n}$ for $N=16$ when $\left\{b_{l} \neq 0, \mathcal{G} \neq 0\right\},\left\{b_{l}=0, \mathcal{G} \neq 0\right\}$, and $\left\{b_{l} \neq 0, \mathcal{G}=0\right\}$.

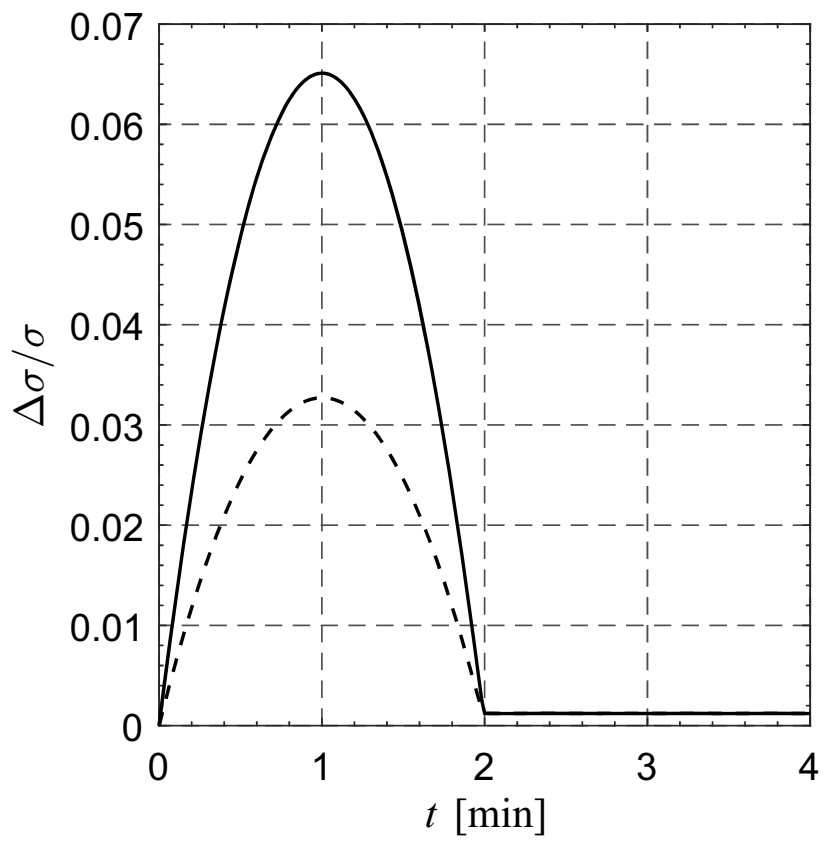

Figure 12: Time evolution of $\Delta \sigma / \sigma$ during attitude maneuver when $N=16$ (solid line) and $N=32$ (dotted line).

[20] P. Janhunen, S. Merikallio, M. Paton, EMMI - Electric solar wind sail facilitated Manned Mars Initiative, Acta Astronautica 113 (2015) 22-28, doi: 10.1016/j.actaastro.2015.03.029.

[21] P. Janhunen, P. K. Toivanen, Safety criteria for flying E-sail through solar eclipse, Acta Astronautica 114 (2015) 1-5, doi: 10.1016/j.actaastro.2015.04.006. 
[22] G. Mengali, A. A. Quarta, P. Janhunen, Considerations of electric sailcraft trajectory design, Journal of the British Interplanetary Society 61 (8) (2008) 326-329 .

[23] A. A. Quarta, G. Mengali, Electric sail mission analysis for outer solar system exploration, Journal of Guidance, Control, and Dynamics 33 (3) (2010) 740-755, doi: 10.2514/1.47006.

[24] P. Janhunen, J.-P. Lebreton, S. Merikallio, M. Paton, G. Mengali, A. A. Quarta, Fast E-sail Uranus entry probe mission, Planetary and Space Science 104 (2014) 141-146, doi: 10.1016/j.pss.2014.08.004.

[25] M. Bassetto, G. Mengali, A. A. Quarta, Attitude dynamics of an electric sail model with a realistic shape, Acta Astronautica 159 (2019) 250-257, doi: 10.1016/j.actaastro.2019.03.064.

[26] P. Janhunen, Photonic spin control for solar wind electric sail, Acta Astronautica 83 (2013) 85-90, doi: 10.1016/j.actaastro.2012.10.017.

[27] P. K. Toivanen, P. Janhunen, Spin plane control and thrust vectoring of electric solar wind sail, Journal of Propulsion and Power 29 (1) (2013) 178-185, doi: 10.2514/1.B34330.

[28] P. K. Toivanen, P. Janhunen, J. Envall, Electric sail control mode for amplified transverse thrust, Acta Astronautica 106 (2015) 111-119, doi: 10.1016/j.actaastro.2014.10.031.

[29] P. Janhunen, P. K. Toivanen, A scheme for controlling the E-sail's spin rate by the E-sail effect itself, in: Space Propulsion 2018, Seville, Spain, 2018

[30] H. Seppänen, T. Rauhala, S. Kiprich, J. Ukkonen, M. Simonsson, R. Kurppa, P. Janhunen, E. Hæggström, One kilometer (1 $\mathrm{km}$ ) electric solar wind sail tether produced automatically, Review of Scientific Instruments 84 (9), doi: 10.1063/1.4819795.

[31] P. K. Toivanen, P. Janhunen, Thrust vectoring of an electric solar wind sail with a realistic sail shape, Acta Astronautica 131 (2017) 145-151, doi: 10.1016/j.actaastro.2016.11.027.

[32] J. R. Wertz (Ed.), Spacecraft Attitude Determination and Control, 1st Edition, Astrophysics and Space Science Library, Springer Netherlands, Dordrecht, Holland, 1978, pp. 760-766. 